\title{
The Myth of WebEDI
}

\author{
Roman Beck, Tim Weitzel, and Wolfgang König \\ Institute of Information Systems \\ Research Project IT Standards and Network Effects \\ J. W. Goethe University \\ Mertonstr. 17, 60054 Frankfurt am Main, Germany \\ Telephone: + $4969798-28273$ \\ Fax: $+4969798-28585$ \\ \{rbeck|tweitzel|wkoenig\}@wiwi.uni-frankfurt.de
}

\begin{abstract}
In this paper, based on empirical research from the German office supply industry principle obstacles to integrating SMEs into EDI networks are identified. Contrary to common belief, integrating SMEs is not so much a technological challenge. We show that due to the fact that many SMEs do not employ e.g. automated material management systems, there are not too many unexploited network gains to be internalized. It is demonstrated that possible solutions need to go beyond what "WebEDI" can offer.
\end{abstract}

Keywords EDI, WebEDI, SME

\section{INTRODUCTION}

Very similar to EDI in the 1960s, the advent of Web technology had many hope for seamless business integration throughout value chains. Especially small and medium sized enterprises (SME) were considered to finally participate in existing EDI networks. But there are drawbacks to SME integration. In this paper, based on empirical research we show that there are principles obstacles to integrating SME requiring coordination designs like ASP-EDI that substantially differ from those often used. It is demonstrated that due to the fact that many

\footnotetext{
The original version of this chapter was revised: The copyright line was incorrect. This has been corrected. The Erratum to this chapter is available at DOI: 10.1007/978-0-387-35617-4_48 
SMEs do not employ e.g. automated material management systems (MMS) there are not too many unexploited network gains to be internalized. The results presented in this paper have been implemented as part of the new Internet strategy within the German office supply industry.

This work is based upon an empirical survey that we conducted in the summer of 2000 about the use of different forms of EDI in the German office supply industry, $95 \%$ of which are SME with less than $€ 1$ million annual revenues. Though dominated by SMEs, this is one of quite few industries that have still found ways of conducting electronic business to business communication which makes it especially appropriate for the analysis.

The structure of the paper is as follows: After a short overview of the evolution of EDI in section 2 the results of the empirical survey are presented. First, in section 3 answers of the (small) retailers and (medium sized) wholesalers and producers to questions concerning reasons for and against EDI, amount of EDI traffic, and Internet usage are used to give an impression about the reality of EDI within SMEs. Then, in section 4, the data is used to derive an individualized profitability analysis concerning EDI and WebEDI. It becomes clear that even rebates on electronic orders cannot compensate SMEs for higher process costs. Finally, in section 5 we propose possible solutions. Especially ASP-EDI offers some advantages to overcome frequent integration problems of SME and could help accelerate the pace of integration.

\section{FROM EDI TO WEBEDI}

\subsection{Traditional EDI}

EDI has been used for over 30 years for the exchange of business data (e.g. delivery notes, invoices) between two application systems in a standardized, automated form (Emmelhainz 1993). EDI solutions are used by companies to achieve a more efficient data and information management by reducing processing time and avoiding redundant data entry. For the benefits associated with traditional EDI (e.g. cost reductions induced by rationalization and automation, shorter order processing time) see (Emmelhainz 1993), (Niggl 1997). Besides the alleged benefits, EDI is not as widespread as many had expected. Presumably, nowadays only $5 \%$ of all companies who could benefit from EDI actually use it (Segev et al. 1997). An important reason is considerably high costs for implementing EDI systems and the lack of MMS (Swatman et al., 1997). Due to 
the fact that many SMEs do not employ MMS, there are not too many unexploited network gains to be internalizes. To earlier (mostly empirical) contributions to the literature on EDI see (Deutsch 1994), (Kilian et al., 1994), (Picot et al., 1993), for WebEDI see (section 2.2) (Curtis 1996), (Densmore 1998) and (Waltner 1997).

\subsection{WebEDI to the rescue?}

The integration of SMEs into value chains is often insufficient. While potential (aggregate) efficiency gains are significant throughout most value chains, not all players are willing to play. As with traditional EDI in the past decades, discrepancies in the technological and organizational way enterprises conduct their business prevent a network-wide adoption of commonly agreed upon standards. Accordingly, many electronic market places have recently faced serious problems due to inadequate process designs and standards. The use of Internetbased EDI solutions or WebEDI has increasingly been discussed especially as a solution to the problem of high EDI implementation and operating costs. First, of course, there is the ubiquitous Internet that lets enterprises replace expensive Value Added Networks (VANs) with a communication medium that is almost free ("EDI over the Internet"). Then, using Web forms allows small enterprises to communicate with their large partners over the Internet while in the background - on the server - all entries are converted to some EDI format required by the large partner ("WebEDI") (Weitzel et al. 2001); for WebEDI benefits among larger partners see (Westarp et al. 1999). Thus, the promise of WebEDI is the low cost and small effort integration of even smallest partners of a value chain basically as long as they have a modem. In fact, WebEDI applications are regarded as a solution to the so-called EDI-dilemma by some authors. But it is important to note that most WebEDI approaches are mostly no more than an HTML-front-end to a shopping system. A user manually enters data into a form using a web browser as communication interface. Thus, there is no machine-tomachine communication (Beck et al., 2002). Another problem rarely discussed in the EDI literature is that many SMEs simply do not want to use WebEDI plainly because it does not pay. We discuss this and further technological prerequisites in the following sections. 


\section{EDI DATA FROM SME}

In the summer of 2000 we conducted an empirical survey with the German office supply industry that consists to up to $95 \%$ of SMEs (section 3.1). Due to this structure comparably early and innovative efforts in EDI standardization are especially appropriate for gathering figures and learning of experiences of small enterprise standardization. Probably, one of the reasons why EDI diffusion stayed below initially expected levels even in large industries known for deep integration like the automotive industry is that SMEs have not been involved appropriately. After a short overview of the office supply industry we present a summary of the results from both market sides, (small) retailers and (larger) producers. The sample is rather small especially for the profitability analysis in the next section, but we could also incorporate the experiences of one author who worked in that area for ten years. Since the typical business process in focus is material management (purchasing) the results are most probably valid for small players in most other industries as well. The overall goal is to get a deeper understanding of the problems inhibiting extensive integration from the perspective of smaller players that are often overlooked. This will aid in finding more adequate technical and organizational solution designs.

\subsection{The German Office Supply Industry}

The German office supply industry is a relatively small part of the retail/trade industry in Germany. Size and market structure make the industry not an attractive target for traditional EDI. Most market participants are small retailers and quite typical SMEs. $88.7 \%$ of all enterprises in that industry (i.e. 8,079 out of $9,104)$ have annual revenues below $€ 0.5$ million. Market volume in 1999 was about $€ 7.9$ billion.

A share of more than 95\% SMEs (i.e. revenues below $€ 1$ million) on the buyer side (retailers) have electronic communication appear as a difficult goal. In the industrial sector there are more than 9,000 retailers and approx. 180 suppliers. The survey was sent to 223 retailers ( $2.5 \%$ of the industry in Germany). 34 answered $(15.25 \%)$. A similar survey was sent to 66 producers and wholesalers (40\% of German industry) 11 (16.18\%) of whom answered. The office supply industry has long been trying to integrate its members. Most notably, an industry consortium called PBS Networks offered a traditional EDI solution ("PBSeasy direct") and a Web-based EDI solution ("PBSeasy online") to address the SME integration problem. All responding retailers therefore use some form 
of EDI ("EDI" or "WebEDI") as described in section 4 but $50 \%$ do not even have automated tracking of outgoing materials.

\subsection{Results from the retailer survey}

In the office supply industry a large heterogeneity of material management systems with more than 13 different solutions are nearly equal implementate on the side of retailers.

This heterogeneity is one of the major obstacles for a common EDI solution (or an underlying unique data model). Most of the WebEDI users do not employ any MMS at all (section 4.2).

We also asked the respondents about the reasons for implementing EDI. The most interesting result compared to traditional literature is that pressure from business partners is comparatively irrelevant (not important, 57\%). The reason is that fewer customers use EDI themselves. Later it will be shown that especially the important goal of cost savings could not be achieved by using EDI.

In addition, we asked for possible reasons against using EDI. One third responded that deals made by traveling salesmen are not describable using EDI. The reasons range from individual pricing negotiations over negotiations not representable in prices at all to contracts outside the scope of EDI as e.g. natural rebates in the form of particular display positions of products in stores.

To a large extent, business process improvements and cost savings depend on the number of partners (and the associated share of EDI messages) also using EDI. At the same time, the number of business documents limits the potential savings. The SMEs of the survey responded to generate on average 3.56 purchase orders per day.

More than a third use EDI for less than $10 \%$ of their purchase orders. The total number of orders and the fraction of electronic orders appear to be too small to benefit from using EDI. As a consequence, those already using EDI stand little chance of increasing the fraction of electronic orders. 

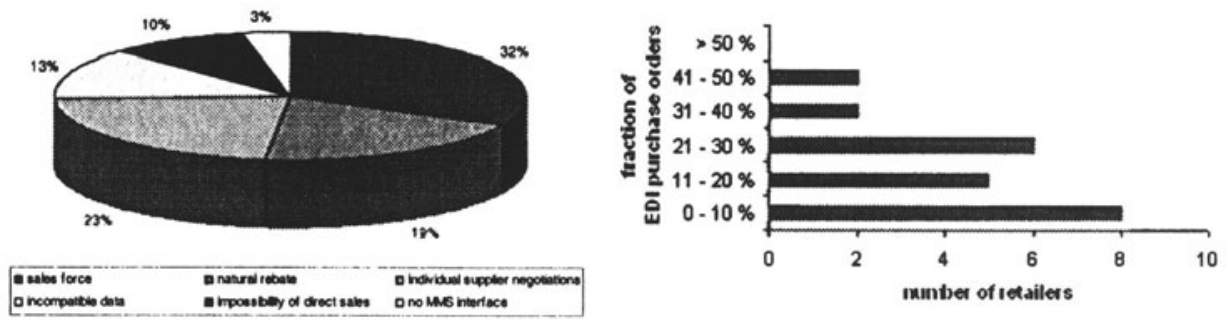

Figure 1: Reasons against implementing EDI among SMEs (left) and fraction of EDI orders (right, excluding WebEDI users)

On average, retailers in our survey have 146.5 communication partners. Out of those, only $9.18 \%$ use EDI. When asked about Internet-based EDI, 30\% of the respondents see advantages compared to X. 400 .

\subsection{Results from the producers and wholesalers survey}

Producers and wholesalers were also asked about their use of EDI and MMS standards. Since this group consists of larger players some used standard ERP systems (more than half SAP R/3). Using SAP systems bears the advantage of being able to use certified EDI converters making implementation and maintenance of EDI systems easier and improving internal data quality.

The producers claim that $0.94 \%$ of all listed retailers are EDI capable. They account for 9.54 of total revenues. This again emphasizes the findings that EDI traffic is found between larger partners. Still, over $80 \%$ of all revenues are generated by business with small enterprises that do not employ any kind of EDI. Revenues generated by EDI derive from an average of 26 electronic orders per day. For $40 \%$ of the respondents, the fraction of EDI orders is less than $1 \%$ of all orders. Only one out of five of the producers execute more than $5 \%$ of all orders via EDI. It is this group that could most probably in fact benefit from the use of EDI.

Since the cost side is regularly easier to quantify than the benefits of standards, only few enterprises made explicit statements to the cost/benefit-ratio. But 2 of 3 considered EDI too expensive to use now although that might change in the future. When asked about reasons for a particular EDI solution the overall picture was that a solution that will be used in the future, too, is highly valued. Over $50 \%$ considered the future availability and use of a solution "very important". 
EDI set-up and operations costs are expectedly higher than for the smaller retailers. Set-up costs consist of costs for the converter ( $€ 18,660$ ), training ( $€$ $13,290)$, and external services ( $€ 29,650)$. Annual operating costs are costs for personnel ( $€ 25,440)$, communication $(€ 7,410)$, and EDI service providers ( $€$ 22,500).

\section{EDI PROFITABILITY ANALYSIS FOR SME}

In the course of our talking to SMEs and as reflected in the figures of the last section we found a higher willingness to standardize among SMEs in the German office supply retail industry than expected but at the same time a lack of human resources and a small factual number of EDI capable messages like orders. On the other hand, participating in automated business processes requires e.g. using MMS to gain net profits from EDI standardization. In this context, the heterogeneity of systems, often individually built, adds to the list of obstacles in the way of standardized messages exchange.

After the aggregate analysis in section 3 we now turn to developing an individual view at the standardization problem as a basis for deriving solution proposals. Therefore, we will use the data from the survey to compare different methods (traditional and web-based EDI) and associated costs of sending order messages to industry partners. The analysis is based upon the EDI solutions PBSeasy direct and PBSeasy online as used in the office supply industry. As profitability benchmark the status quo of sending orders by FAX is used. It turns out that for an average frequency of 4 orders per day a breakeven for neither EDI solution can be achieved. The most efficient alternative to EDI that is actually in use is ordering by FAX.

\subsection{Traditional EDI for SME}

The analysis is based on the following premises concerning the traditional EDI solution PBS easy direct. A typical German working week consists of 37.5 person hours (PH) (230 days per year). An EDI administrator costs $20 € / \mathrm{PH}$. Due to special agreements with Seeburger, the EDI converter (WinELKE) is quite inexpensive for the SMEs. A Win95 version costs about $€ 1,990$. Other costs include integration with the MMS and installation costs of $€ 75$ for a telebox 400 (German Telekom). The planning horizon is 5 years with $4 \%$ interest for the linear depreciation of the converter. System administration is assumed to 
take 30 days per year according to our survey and the literature (Klagge et al. 1998). Annual maintenance costs for Seeburger are $15 \%$ of the license costs.

In our survey, there is an average of four orders per day (ranging from 0 to 20). No SME used EDI for communication with his customers, thus it is only outgoing orders that are of interest here. Corresponding to the survey the SME assumes a digressive growth of EDI traffic for the following five years (from $25 \%$ to $10 \%$ p.a.). The EDI communication costs depend on a fixed connectivity price for phone (0.06 €/unit) and telebox 400 access $(0.11 € / \mathrm{min})$ and furthermore a data transmission based pricing depending on the transferred $\mathrm{KBs}$ from the 1st unit of $2 \mathrm{~KB}$ ( $€ 0.11$ ) to the 50th unit ( $€ 0.0003)$. One typical order therefore is at least $€ 0.26$. Multiple orders can be sent simultaneously thus economizing on the access fee. Other costs include ILN registration (international location number, necessary condition for EANCOM (i.e. EDIFACT subset) participation) ( $€ 61.32$ p.a.) and a $€ 38.35$ per month fee to PBS network.

In contrast, conventional pre-EDI ordering was done by FAX so we will compare the EDI and WebEDI solution with this method as benchmark in section 4.3. Traditional orders induce costs of paper, printing, and FAX. The costs of ca. $0.65 € /$ message cover all these and communication costs. Experience shows that the whole process takes approximately 3 minutes. As we will see personnel costs are the decisive factor using FAX instead of EDI with associated high maintenance costs for the EDI converter software administration. This is an indication that IT qualification for smoothly running the system is a critical success factor rather than the initial setup costs for the converter itself. As we will show in section 4.3 parts of the EDI-dilemma can be solved if the industry partners enjoying the most benefits from EDI are willing to share (and therefore enable) a part of those benefits.

\subsection{WebEDI for SME}

In the following efficiency analysis we assume that both, a PC and an Internet modem as well as Internet access are already available and are used not exclusively for the WebEDI application via Internet so that no depreciation needs to be considered. Furthermore, online fees of Deutsche Telekom, Germanys largest connectivity provider, were used for the calculation. The Web-based PBSeasy online is considered to economize on costs using inexpensive and flexible Internet technology.

Gathering order data is equivalent to the conventional way described above and takes approximately 25 minutes. Additionally, sending the FAX and finding list prices takes 3 minutes, using WebEDI 10 minutes for stuffing the shopping 
cart. PBSeasy online fees are $20 € /$ month plus $4 € /$ month for German Telekom that compensate for the first 12 orders (at 10 minutes each).

The advantages for the specialized office supply retailers, however, are strongly reduced due to the manual entry and dispatching of the orders. Without a functioning material management system automating this process ordering over the Internet even induces an additional workload. So far, the retailer was able to send his order immediately after writing the FAX. Now, he has to manually enter order data a second time, this time into the Internet form.

A disadvantage is to be seen in the field of processing and communication time. WebEDI is more time consuming than a FAX transaction resulting in higher personnel and communication costs. Additionally, not all relevant industry partners are part of the WebEDI solution inducing additional work again. Further, some individual needs of the SMEs are not sufficiently addressed compared to ordering from the traveling salesman (see Figure 1). Another important disadvantage associated with the repeated manual entry process into web forms is an increased risk of errors that is often underestimated.

This brief discussion shows that technologically approaching the EDI integration problem with SMEs by means of WebEDI cannot always provide sufficient incentives to standardize. As a consequence, additional organizational means could be used to solve this problem. As a consequence, the provider of the market place (i.e. PBSeasy online) established a system of vertical utility redistribution in a way that monetary transfers should compensate SMEs for the additional expenditure. Since SMEs using WebEDI typically do not employ automated MMSs it must be assumed that data entry e.g. for orders implies material costs as well as additional personnel expenditures (in accordance with the survey at average costs of $20 \mathrm{E} / \mathrm{h}$ ). The cash bonus model in the industry's WebEDI case offers a $1 \%$ incentive of the purchase order value (maximum of $€ 50$ per month) for the WebEDI user. The data show that WebEDI represents no overall alternative to conventional inter-company business transactions per se.

\subsection{EDI vs. WebEDI vs. FAX}

Corresponding to our survey the average number of orders sent via EDI was 920 in 2000 . Due to the expected rise in numbers we assumed a decreasing growth ratio from 920 in 2000 to 1,746 in 2004.

In Table 1 the costs development in the different scenarios for SMEs based on the figures presented above is shown. All EDI based services are compared to the most common way of ordering, i.e. by FAX. For FAX transmission the manually created order will be sent by hand to the industry partner. This process 
takes approx. 3 minutes inducing connectivity and personnel costs. For the communication we determined costs of $€ 0.18$ per order which is equivalent to 3 minutes connection in telephone prices in 2000 . The personnel costs are $€ 10$ per hour as identified earlier. The material costs ( $€ 0.06$ per order) are the same as for WebEDI.

For EDI based ordering there are no material and personnel costs for each order but other costs include setup and system administration for the converter software. The SMEs in the office supply industry have a special offer from Seeburger for the WinELKE converter about $€ 1,995$. This software can be linearly depreciated with $€ 414.15$ p.a. Further costs for updates and maintenance amount to $€ 299.10$ per year. The higher qualification requirements for a system administrator imply a higher wage of $20 € / \mathrm{h}$ (see above) independent of the amount of orders.

According to the survey, an EDI administrator is needed for about 30 working-days which causes $€ 4,600$ p.a. Furthermore, for using PBSeasy direct and the ILN number for identification within the EANCOM EDI standard costs of $€$ 521.52 arise.

Besides the licensing fees and communication costs WebEDI causes a considerable increase in personnel costs due to the fact that it takes the ordering party approximately ten minutes longer to supply and send their orders when using a web interface.

\begin{tabular}{|c|c|c|c|c|c|}
\hline \multirow{3}{*}{$\begin{array}{l}\text { year } \\
\text { growth rate } \\
\text { orders }\end{array}$} & 2000 & 2001 & 2002 & 2003 & 2004 \\
\hline & & 0.25 & 0.2 & 0.15 & 0.1 \\
\hline & 920 & 1150 & 1380 & 1587 & 1746 \\
\hline \multicolumn{6}{|l|}{ conventional (FAX) } \\
\hline & $€$ & $€$ & $€$ & $€$ & $€$ \\
\hline material & 56.45 & 70.56 & 84.67 & 97.37 & 107.11 \\
\hline communication & 169.34 & 211.68 & 254.01 & 290.48 & 321.38 \\
\hline processing & 940.78 & $1,175.97$ & $1,411.17$ & $1,622.84$ & $1,785.43$ \\
\hline system administra- & 0.00 & 0.00 & 0.00 & 0.00 & 0.00 \\
\hline maintenance & 0.00 & 0.00 & 0.00 & 0.00 & 0.00 \\
\hline depreciation & 0.00 & 0.00 & 0.00 & 0.00 & 0.00 \\
\hline
\end{tabular}




\begin{tabular}{|l|l|l|l|l|l|l|}
\hline SUM & $\mathbf{1 , 1 6 6 . 5 7}$ & $\mathbf{1 , 4 5 8 . 2 0}$ & $\mathbf{1 , 7 4 9 . 8 5}$ & $\mathbf{2 , 0 1 0 . 6 9}$ & $\mathbf{2 , 2 1 3 . 9 2}$ \\
\hline EDI \\
\hline material & 0.00 & 0.00 & 0.00 & 0.00 & 0.00 \\
\hline communication & 244.60 & 305.75 & 366.90 & 421.94 & 464.21 \\
\hline processing & 0.00 & 0.00 & 0.00 & 0.00 & 0.00 \\
\hline system administra- & $4,600.00$ & $4,600.00$ & $4,600.00$ & $4,600.00$ & $4,600.00$ \\
\hline maintenance & 299.10 & 299.10 & 299.10 & 299.10 & 299.10 \\
\hline depreciation & 414.15 & 414.15 & 414.15 & 414.15 & 414.15 \\
\hline ILN and PBSeasy & 521.52 & 521.52 & 521.52 & 521.52 & 521.52 \\
\hline SUM & $\mathbf{6 , 0 7 9 . 3 7}$ & $\mathbf{6 , 1 4 0 . 5 2}$ & $\mathbf{6 , 2 0 1 . 6 7}$ & $\mathbf{6 , 2 5 6 . 7 1}$ & $\mathbf{6 , 2 9 8 . 9 8}$ \\
\hline WebEDI & & & & & \\
\hline material & 56.45 & 70.56 & 84.67 & 97.37 & 107.11 \\
\hline communication & 261.36 & 339.48 & 417.09 & 486.94 & 540.60 \\
\hline processing & 1.567 .96 & $1,959.78$ & 2.351 .94 & $2,704.73$ & 2.975 .72 \\
\hline system administra- & 0.00 & 0.00 & 0.00 & 0.00 & 0.00 \\
\hline maintenance & 0.00 & 0.00 & 0.00 & 0.00 & 0.00 \\
\hline depreciation & 0.00 & 0.00 & 0.00 & 0.00 & 0.00 \\
\hline Telekom,PBSeasy & 294.50 & 294.50 & 294.50 & 294.50 & 294.50 \\
\hline SUM & $\mathbf{2 , 1 8 0 . 2 7}$ & $\mathbf{2 , 6 6 4 . 3 2}$ & $\mathbf{3 , 1 4 8 . 2 0}$ & $\mathbf{3 , 5 8 2 . 5 4}$ & $\mathbf{3 , 9 1 7 . 9 3}$ \\
\hline
\end{tabular}

Table 1: FAX vs. EDI vs. WebEDI

Under the premises outlined above there is a clear dominant relation between the scenarios in Table 1. From the individual perspective of the SME it is always a dominant strategy to use FAX regardless of the number of orders. At the same time, unexploited network gains are evident, too, indicated by the obvious preference of the industry partners towards EDI orders. The development shown in Table 1 is depicted in Figure 2 (left). The order curve reflects the expected increasing number of orders (scaled on the second ordinate). On the left ordinate the total costs for each order dispatching method are shown. Correlated with the increasing order curve the WebEDI and FAX curves increase over time while the cost development of EDI shows smaller sensitivity to the number of orders. At constant prices for EDI and WebEDI those curves show no intersection in the next few years.

The German office supply industry offers two structurally different bonus models for EDI and WebEDI. For the traditional EDI solution there is a bonus of $€ 5$ for the first 500 orders each, $€ 2.50$ for orders $501-1,000$, and $€ 1$ for orders 
number 1,001-1500. For the WebEDI bonus model see section 4.2. Using the bonus models as a response to the particular cost situation of SMEs has the situation change. The diagram on the right of Figure 2 shows the implications of the two bonus models intended to make WebEDI and EDI more attractive for SMEs.
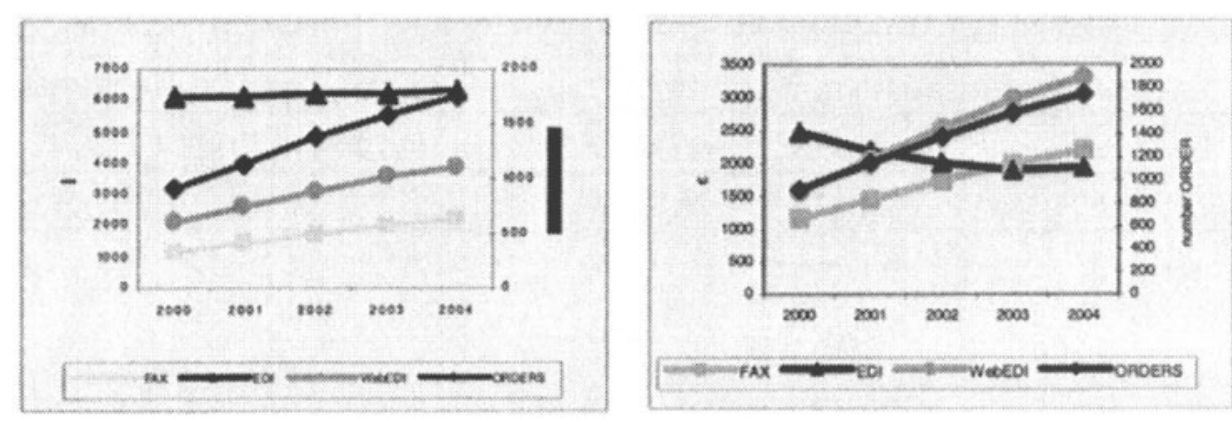

Figure 2: FAX vs. EDI vs. WebEDI cost development without (left) and with (right) subsidies

As the right diagram in Figure 2 shows, the compensation is too small to make WebEDI more desirable than a FAX (mostly due to the limited refund of $€$ 50 per month). The higher gradient of the WebEDI curve compared to the order (and FAX) curve sketches this. The curve is still steeper than the FAX cost development curve. Thus, despite the existing refund mechanism FAX is still always preferable from an SME's point of view.

The subsidization model for EDI is more consequent and more efficient and is, in spite of WebEDI, profitable to use. With the refunds conditions offered in 2000 an SME could work efficiently with an EDI converter when the number of orders is higher than 1,475 or the costs appearing ordering by FAX are higher than $€ 1,957.56$ p.a.

Interestingly, the WebEDI solution is the most expensive solution. With more than 1,068 orders or $€ 1,889.68$ processing costs it is better to use an EDI converter system than WebEDI and from 1,474 orders the EDI system is absolutely the least expensive way to order. Of course, the economic effect for SMEs depends on the offered refunds but from an economic point of view an automated order system with less intensive personnel input is most probably the more efficient solution from an aggregate perspective.

Apart from the isolated view at the business transaction where the economic effect for SMEs depends on the offered refunds, real integration requires system to system communication. Thus, from an aggregate perspective, establishing 
"real" EDI relations, i.e. mutually integrated processes, requires all parties to have MMSs the benefits from which may considerably exceed those of the infamous WebEDI scenarios.

\section{CONCLUSION}

What does the future hold? The lessons learnt from the survey clearly mirror experiences from a multitude of standardization processes: Aggregate goals that require the participation of various individuals entail the search for a common denominator. In this case, the smallest common - technological - denominator probably is the print spooler inherent in basically all office software. Here, a recent trend often called ASP-EDI tries to incorporate the findings as presented in this paper to find a balance between simplicity on the side of the small and (at least some) functionality on the side of the larger players. The basic idea is sending the order print spool (ASCII) to an intermediate web server customized to the respective MMS converting the data set to the required format and vice versa. Although connectivity costs have been shown to be of no significant importance this approach also offers offline functionality. Of course, a necessary condition for true supply chain integration still is the existence of an MMS. Based upon our findings, first implementations of this approach are offered as PBSeasy Mail (Beck et al., 2002). In a next step, as part of a Web Service scenario this service could be implemented to further overcome the technological side of compatibility problems. Some innovators already offer this sort of service. As a consequence, we consider what is now called WebEDI to be a shortlived phenomenon.

In the future the application of XML/EDI is conceivable in order to map the data at the SME immediately to reduce the costs. This service, too, could be provided by external entities. A generalization of this concept is known as WebService. Web Services have been defined as "encapsulated, loosely coupled contracted functions offered via standard protocols" (www.webservices.org). On an abstract layer, these can be interpreted as autonomous services accessible via the Web that can serve as components of a dynamic value chain. Such WebServices - including converting the EDI print-spool data to standard XML/EDI formats like $\mathrm{xCBL}$ - can be another step towards the integration of the most heterogeneous partners in that they increasingly allow the outsourcing of compatibility problems. Already, XML-based languages for calling, describing, and registering WebServices like XML-Protocol, UDDI and WSDL are available. 
Altogether, it has been shown that there are technological and organizational drawbacks restraining SMEs from participating in EDI networks such as supply chains that have been adapted to the needs and possibilities of larger players who can economize on costs by automating their business processes. In fact, EDI has been proven to be a great source of efficiency for high transaction volume communication. When trying to broaden the range of partners within a value chain that participate in system to system infrastructures it is important to see who actually benefits from such architectures. In order to open the way for smaller players into those networks, there are technical as well as organizational issues that - if they are adequately considered - have the old idea of SMEs being a part of electronic communication appear quite promising.

\section{REFERENCES}

Beck, R., Weitzel, T., König, W. (2002): Promises and pitfalls of SME integration, in: Proceedings of the $15^{\text {th }}$ Bled Electronic Commerce Conference ereality 2002, Bled, Slovenia.

Curtis, C. (1996): EDI over the Internet: Let the games begin, in: Internetweek, Issue 627, September 9,1996, http://www.techweb.com/se/directlink.cgi?CWK19960909S0076.

Densmore, B. (1998): EDI vs. The new kids, in: Computerworld, April 6, 1998, http://www.computerworld.com/home/Emmerce.nsf/All/980406edi

Deutsch, M. (1994): Unternehmenserfolg mit EDI: Strategie und Realisierung des elektronischen Datenaustausches, Braunschweig.

Emmelheinz, M. (1993): EDI - A Total Management Guide, 2nd edition, New York.

Kilian, W., Picot, A., Neuburger, R., Niggl, J., Scholtes, K.-L.Seiler, W. (1994): Electronic Data Interchange, Baden-Baden.

Klagge, D., Nett, W., Windler A. (1998): Electronic Data Interchange im mittelständischen Betrieb, Köln.

Niggl, J. (1994): Die Entstehung von Electronic Data Interchange Standards, Wiesbaden.

Picot, A., Neuburger, R., Niggl, J. (1993): Electronic Data Interchange (EDI) und Lean Management, in: Zeitschrift für Führung und Organisation, Nr. 1/1993, S. 20-25.

Segev, A., Porra, J., Roldan, M. (1997): Internet-Based EDI-Strategy, Universitiy of Berkeley, California.

Swatman, P., Poon, S. (1999): Internet-based Small Business Communication: Seven Australian Cases, in: Electronic Markets, http://www.electronicmarkets.org/netacademy/publications.nsf/all_pk/89/\$file $/ v 7 n 2 \_$poon.pdf?OpenElement\&id $=89$. 
Waltner, C. (1997): EDI Travels The Web - EDI over the Web offers companies cheaper E-commerce and messaging via browsers, in: Internetweek (668), 16-06-1997, http://www.techweb.com/se/directlink.cgi?CWK19970616S0066.

Westarp, F.v., Buxmann, P., Weitzel, T., Koenig, W. (1999): The Status Quo and the Future of EDI, in: Proceedings of the 1999 European Conference on Information Systems (ECIS'99), p. 719 -731.

Weitzel, T., Harder, T., Buxmann, P. (2001): Electronic Business und EDI mit $X M L$, Heidelberg. 